\title{
Difference in response reliability predicted by STRFs in the cochlear nuclei of barn owls
}

Louisa J. Steinberg \& Jose Luis Pena, Department of Neuroscience, Albert Einstein College of Medicine, Bronx, NY

\begin{abstract}
The brainstem auditory pathway is obligatory for all aural information. Brainstem auditory neurons must encode the level and timing of sounds, as well as their time-dependent spectral properties, the fine structure and envelope, which are essential for sound discrimination. This study focused on envelope coding in the two cochlear nuclei of the barn owl, nucleus angularis (NA) and nucleus magnocellularis (Nime (ITD) and level (IID) differences respectively. We found that NA neurons though unable to accurately encode stimulus phase, lock more strongly to the stimulus envelope than NM Units. The spectrotemporal recentive fields (STRFs) of NA newons exhibst a preexcitatory suppressive field. Using multilinear regression analysis and computational modeling, we show that this feature of STRFs can account for enhanced across-trial response reliability, by locking spikes to the stimulus evise envelope coding between the time and intensity processing pathways as early as at the level of the cochlear nuclei. This allows the ILD processing pathway to encode envelope information with greater fidelity than the IrD piocessing pathway. Funthermore, we demonstrote that the properties of the neurons' STRFs can be quantitatively related to spike timing reliability.
(AN
(NA) $\rightarrow$ (ILD) $\rightarrow(1 C 15) \rightarrow(1 C X)$

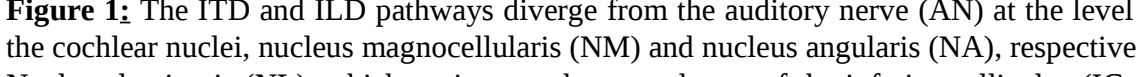

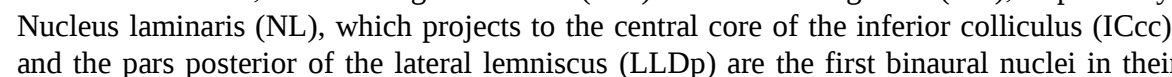

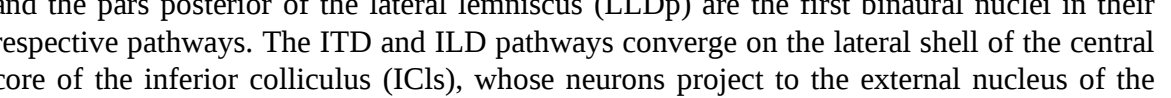
ifferio colliculus (ICX), giving rise to to space-specific neurons and a map of auditiory
\end{abstract}

\section{Methods}

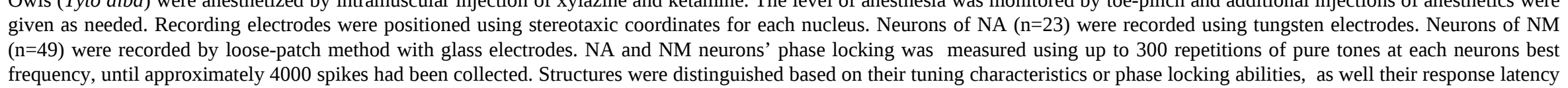

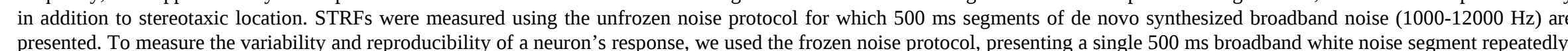
Analysis

\section{Analysis} \section{$\mathrm{g}$
$\mathrm{s}$
$\mathrm{T}$
$\mathrm{s}$
5
$\mathrm{a}$
$\mathrm{c}$
$\mathrm{c}$
$\mathrm{T}$
$\mathrm{x}$
$\mathrm{x}$
$\mathrm{x}$$$
\text { The }
$$

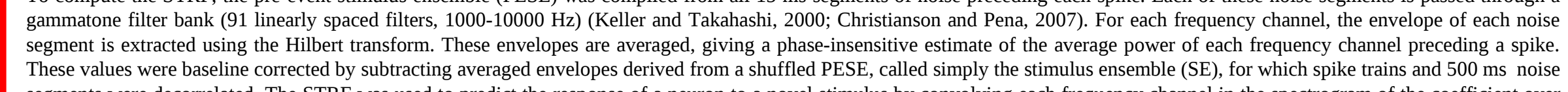

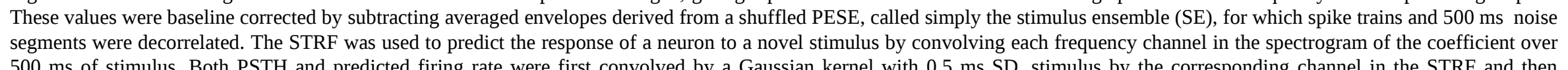

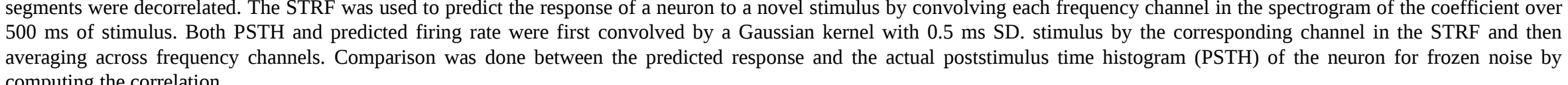

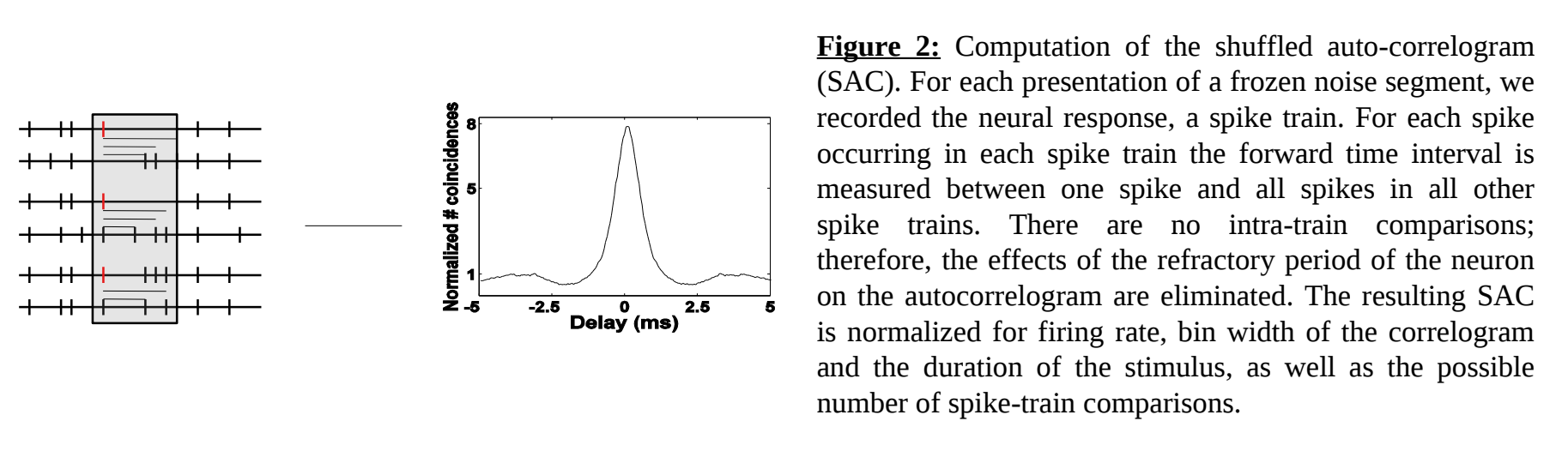

NA and NM units exhibit gross differences in their response properties

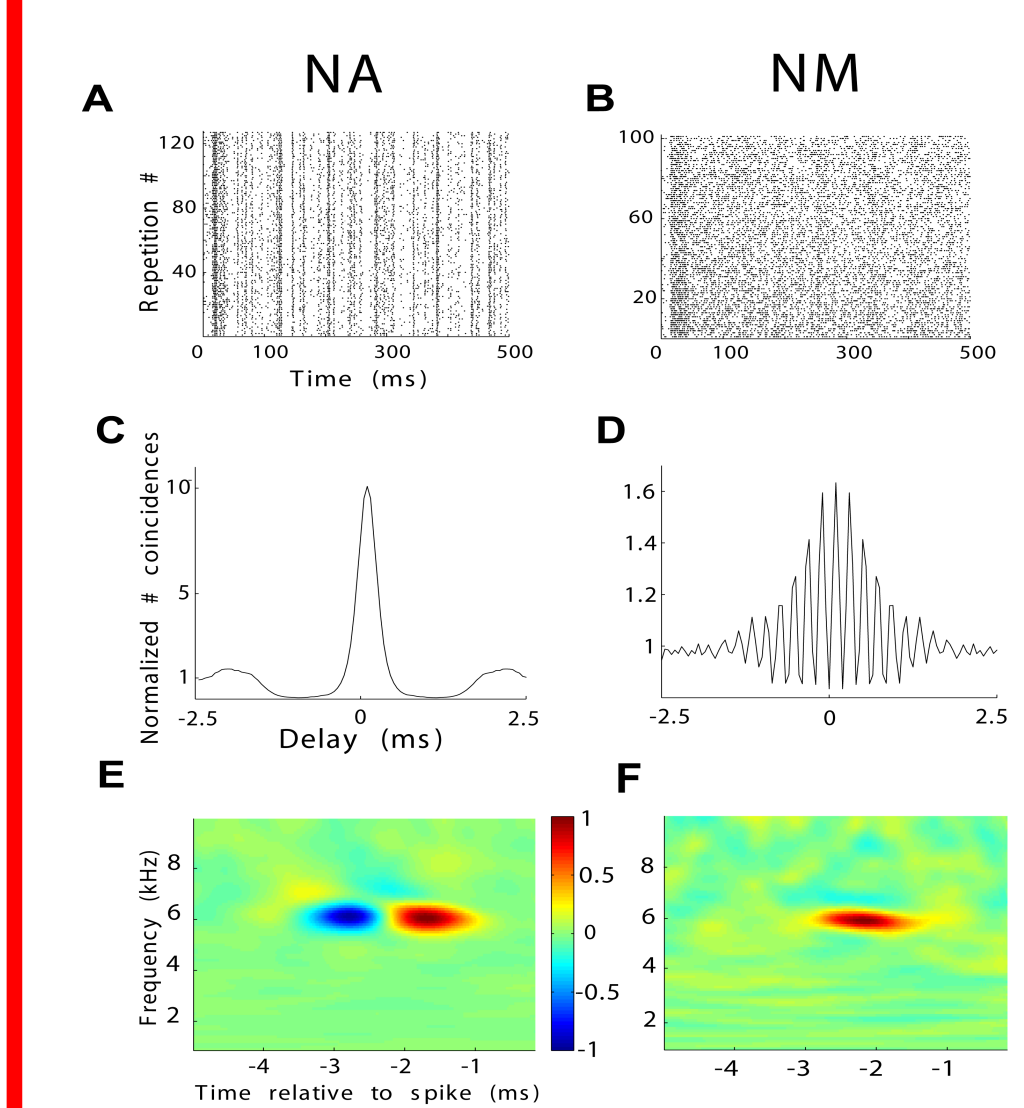

Figure 3: Representative rasters are shown for the responses of NA (A) and NM (B) neurons to repeated presentations of the same stimulus (frozen noise). (C) SAC corresponding to the NA unit in (A) with a peak height of 10 . (D) SAC corresponding to the NM unit in (B) with a peak height of 1.6. The high-frequency structure in $\mathrm{D}$ is a result of phase locking in NM. (E) A representative example of NA unit's STRF. STRFbf = $6.1 \mathrm{kHz}, \mathrm{STRFbw}=800 \mathrm{~Hz}$, STRFtw $=0.9 \mathrm{~ms}$. (F) A representative example of a NM unit's STRF. STRFbf $=$ $6 \mathrm{kHz}, \mathrm{STRFbw}=500 \mathrm{~Hz}$, STRFtw $=0.9 \mathrm{~ms}$. Notice that the NA unit's excitatory subfield is preceded by a suppressive field (blue), which is absent in NM.

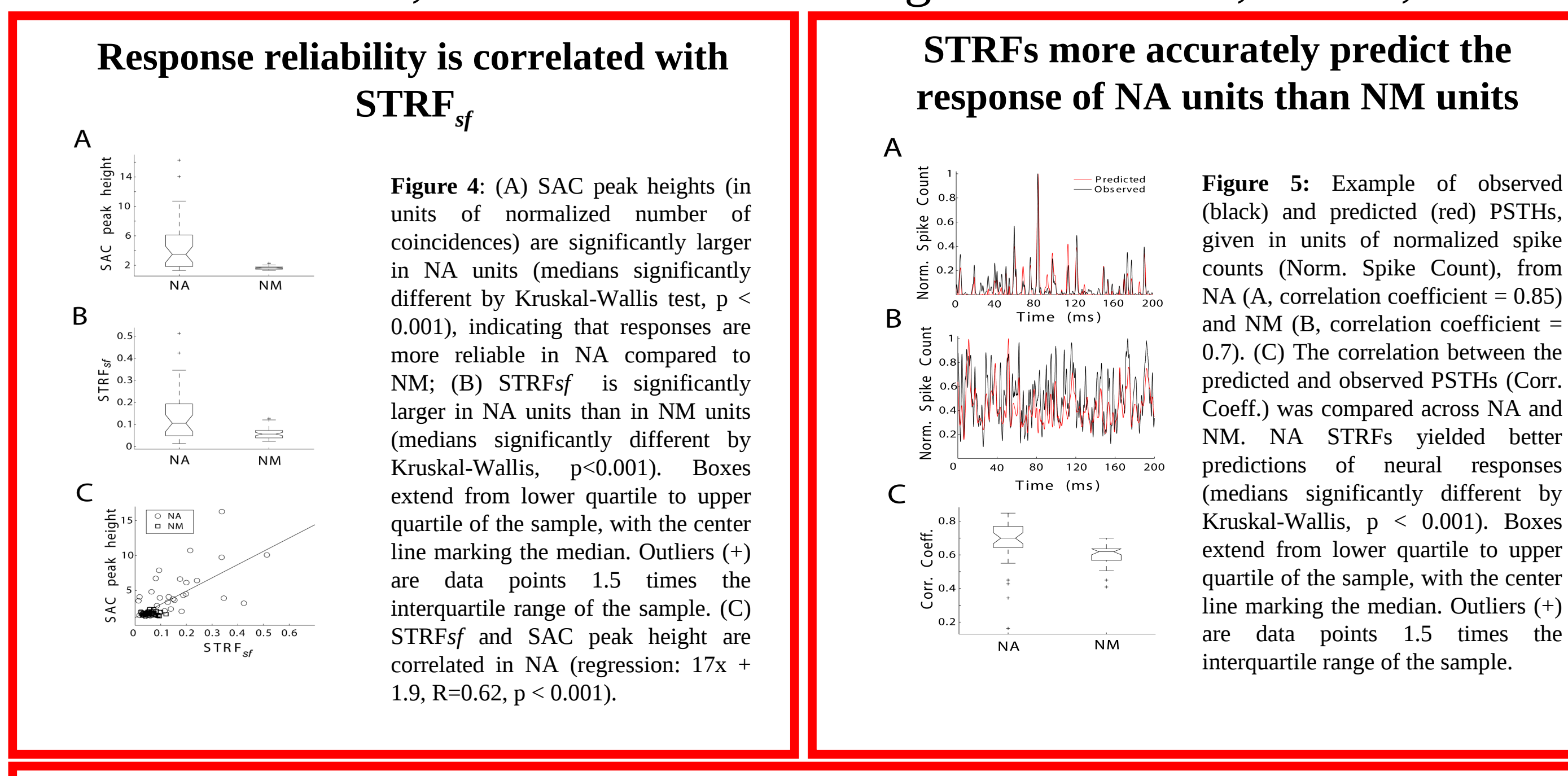

\begin{tabular}{|c|c|c|c|c|c|}
\hline \multicolumn{5}{|c|}{ Coefficients of multilinear regression analysis } & \multirow{2}{*}{$\begin{array}{l}\text { Multilinear regression analysis shows that } \mathrm{STRF}_{s f} \text { is the } \\
\text { greatest contributor to response reliability }\end{array}$} \\
\hline & Reg. ceeff. (NA) & $p(\mathbb{N A})$ & Reg. coeff. (NM) & $p(\mathbb{N M})$ & \\
\hline $\mathrm{STRF}_{\mathrm{H}}$ & 10.16 & $<0.001$ & -0.09 & 0.57 & 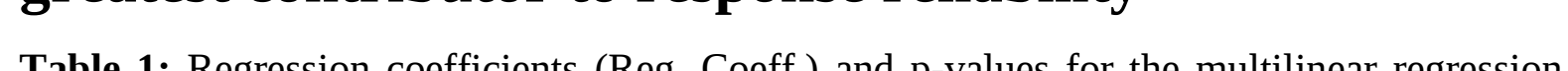 \\
\hline STRFam & 2.57 & 0.28 & 0.17 & 0.57 & $\begin{array}{l}\text { Table 1: Regression coeffficients (RReg. Coeff.) and p-values for the multilinear regeression } \\
\text { analysis performed on STRFS, STRFbw, STRFbf and SAC peak height tata from NA and }\end{array}$ \\
\hline STRF & -7.95 & $<.001$ & -1.4 & $<.001$ & \\
\hline
\end{tabular}

Modeling the effects of $\mathrm{STRF}_{s f}$ on response reliability.

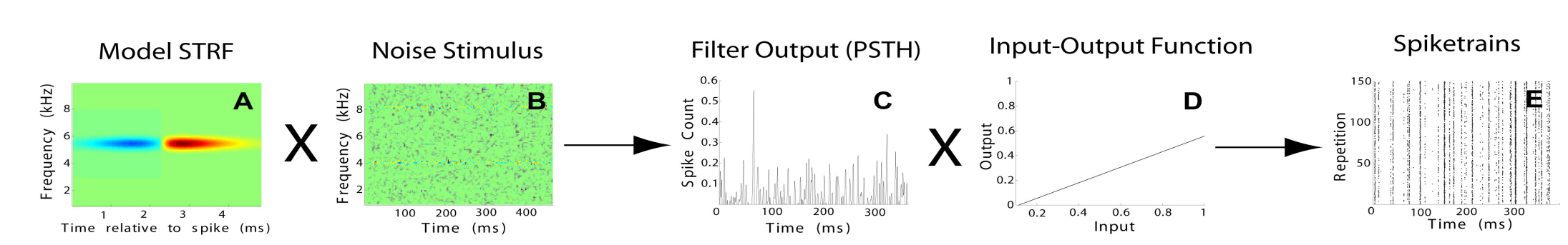

Figure 6: Schematic description of the model. Model STRFs (A) were convolved with a noise stimulus (B). This PSTH-like filter output (C) was put Figure 6: Schematic description of the model. Model STRFs (A) were convolved with a noise stimulus (B). This PSTH-like fiter output (C) was put through a linear input-output

A

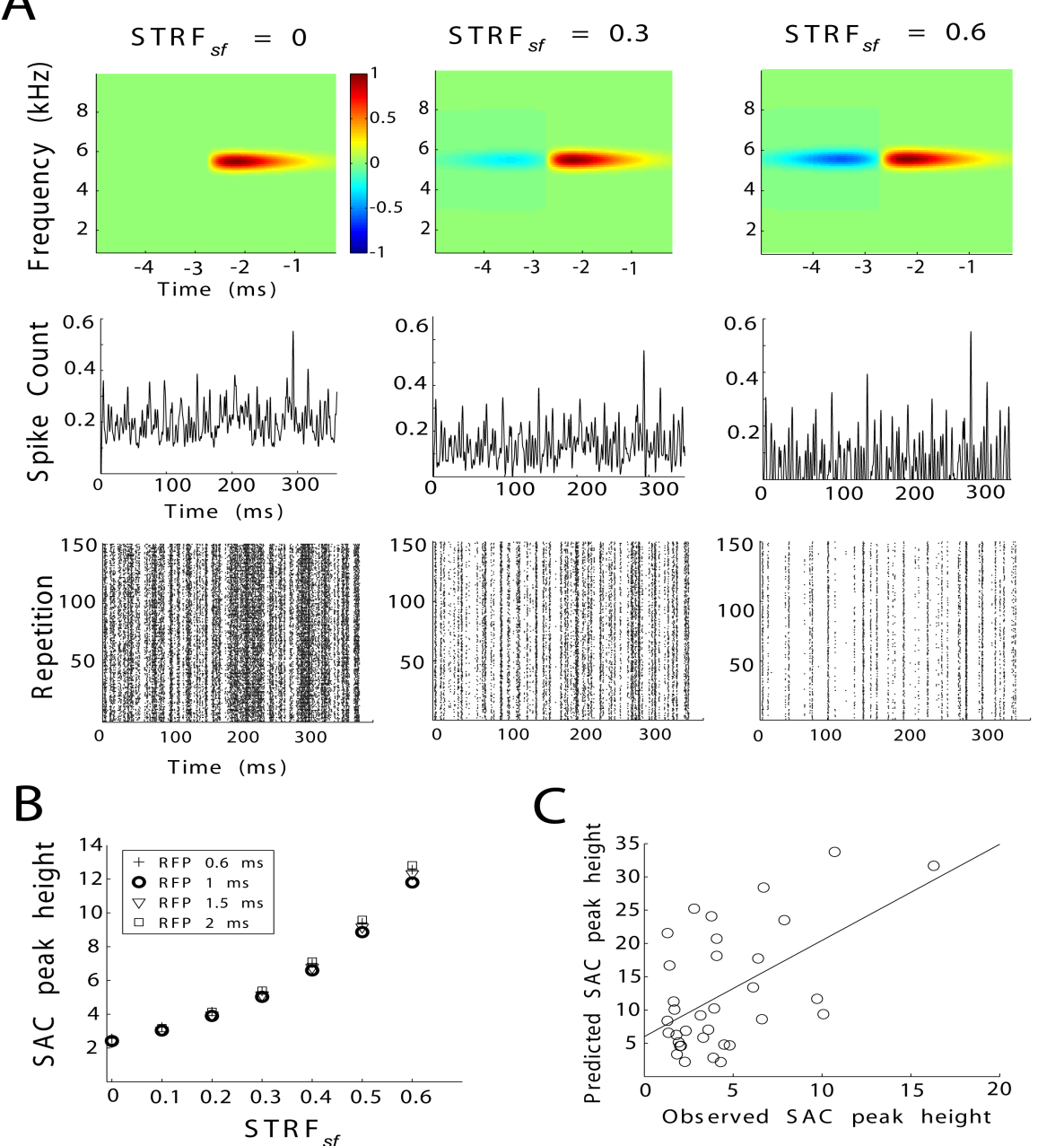

Figure 7: (A) Representative STRFs (top), PSTHs (middle) and corresponding asters (bottom) at suppressive field magnitudes (STRFs $f$ equal to, from left to right, 0.3 , and 0.6. PSTHs are shown as normalized predicted spike count. Quantification periods (RFP) were tested (indicated in B). Datapoints for RFP = $1 \mathrm{~ms}$ are enhanced

Conclusions
- The cochlear nucleus angularis (NA) shows greater envelope
locking than its counterpart in the ITD pathway, nucleus
magnocellularis (NM).
- STRFs in NA showed significantly larger pre-excitatory
suppressive fields than NM, indicating that NA has more
selective spectrotemporal tuning compared to NM.
- Modeling shows that greater pre-excitatory suppressive fields
in the STRF enhance response reproducibility
- The first processing stage of the ILD pathway conveys
spectrotemporal information with greater fidelity than the ITD
pathway
height, B, in units of normalized number of coincidences). Different refractory for clarity. A refractory period of $1 \mathrm{~ms}$ most closely approximates the estimated refractory periods across NA and NM. (C) The SAC peak height predicted by the
model is correlated with the in vivo observed $S A C$ peak height $(R=0.54, p=0.001)$. 